\title{
DEVELOPING DIRECT ACCESS SKILLS IN AUTHENTIC PRIMARY CARE PLACEMENTS
}

Dear Editor

We were pleased to see how Quach et al. ${ }^{1}$ approached the importance of student dental hygienists and dental therapists (DH\&DT) learning to work in direct access (DA). We agree that the majority of primary care dentistry is within the Scope of Practice (SoP) of these professionals and, but for restrictive NHS contracts, they should be ready to provide patient care without a dentist's prescription. However, we were disappointed that it was seen as a 'natural next step to nurture confidence' rather than established practice. After all, DH\&DT qualified post-2002 should have the required training to do this.

At Sheffield we recognised this in 2014 and developed a protocol for senior DH\&DT students to treat patients 'as-if' in direct access. Our dental practice unit, whilst under the governance of a large Foundation Trust, was established in the 1980s to provide students with a primary dental care experience. The majority of patients have been under our care for some years and represent a cohort similar to that of an established general dental practice, except that they are accustomed to being treated by students. This unit gives an environment for DH\&DT students to learn to undertake patient assessment, prescribe and interpret radiographs and develop a treatment plan under the supervision of an experienced dentist clinical teacher. DH\&DT students are additionally expected to identify treatment and potential diagnoses without their SoP and refer to BDS students or secondary care as appropriate.

The protocol has subsequently been adopted by some of our CDS and GDS Outreach practices so that students can develop their DA skills in authentic primary care placements. It is unfortunate that, within both the trust and outreach placements, supervision must be done by a registered dentist, rather than a dental therapist, to satisfy commissioning and governance obligations. However, we are ready to embrace changes to prescribing exemptions so that our DH\&DT students will be able to work more autonomously upon registration and give truly widened access to dental treatment in the community. Emma Bingham and Dr Adrian Jowett, School of Clinical Dentistry, University of Sheffield

\section{Reference}

1. Quach D D, Davda J S, Radford L R et al. Are dental schools doing enough to prepare dental hygiene \& therapy students for direct access? BDJ Team 2020; 7: 36-37. Available at: https:// doi.org/10.1038/s41407-020-0471-1 (accessed January 2021).

\section{FGDP appoints new DCP representative}

The Faculty of General Dental Practice UK (FGDP) has appointed Mrs Sarah Hill BSc RDH FHEA as its new Dental Care Professional Representative, succeeding the late Janet Goodwin.

Sarah qualified as a dental hygienist from the University of Bristol, with distinction, in 2012, and also holds a BSc (Hons) in Biomedical Sciences from the University of Southampton as well as a Postgraduate Diploma in Clinical Education from the University of Plymouth.

Working as a hygienist in a mixed orthodontic practice in Halesowen, where one of her passions is making sure that her patients are at ease during treatment, Sarah also delivers presentations to dental hygiene and therapy students on behalf of TePe. She has previously been a lecturer in Community Based Dentistry at Peninsula Dental School - a role which included clinical supervision, assessing practical and written examinations, and working with students on community projects - and a sales representative for GlaxoSmithKline.

Sarah has been an Affiliate Member of FGDP since 2013, and was recently co-opted onto the board of the Faculty's West Midlands division as its DCP representative. An assessor for the National Examining Board for Dental Nurses, and Fellow of the Higher Education Academy, she is also an active member of the British Society of Dental Hygiene and Therapy, and was Chair of its South West and South Wales group for three years.

FGDP is the only professional membership body in the UK specifically for general dental practice, and reflects and represents individuals across the whole dental team, welcoming all GDC-registered dental therapists, dental hygienists, dental nurses, dental technicians, clinical dental technicians and orthodontic therapists.

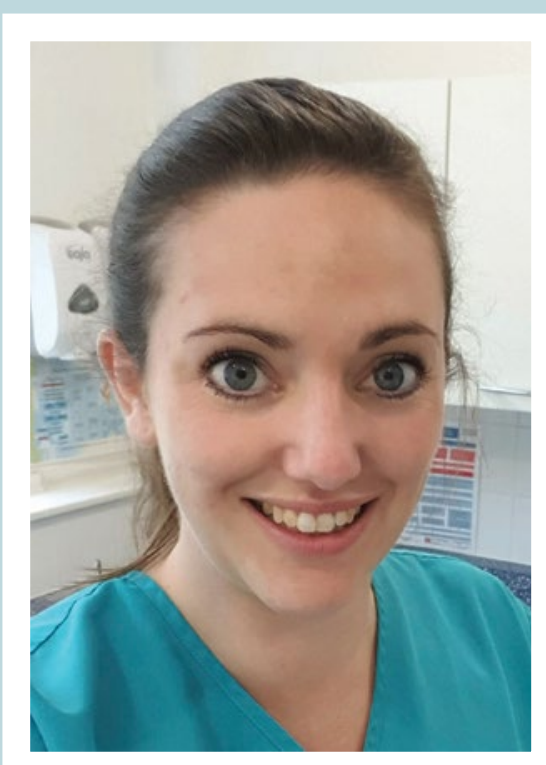

Sarah will represent the interests of the wider dental team at meetings of the National Faculty Board, and in the FGDP's work.

Commenting on her appointment, Sarah said: 'I'm absolutely delighted, and look forward to representing the interests of my colleagues on the Board, and helping the Faculty in its mission to raise standards of dental care for patients. 